\title{
Pflegerische Versorgungsdefizite in deutschen Krankenhäusern - Ergebnisse einer bundesweiten Befragung von Krankenhaus-Leitungspersonen
}

\section{Nursing Care Deficits in German Hospitals - Results of a Nationwide Survey of Supervisory Staff in Hospital}

Autoren

Institut

\author{
A. Reifferscheid, N. Pomorin, J. Wasem
}

Lehrstuhl für Medizinmanagement, Universität Duisburg-Essen, Essen

\author{
Schlüsselwörter \\ - Pflege \\ - Krankenhaus \\ - Rationierung \\ persönliche Zuwendung \\ - Umfrage
}

\section{Key words \\ - nursing \\ - hospital \\ - rationing \\ - personal care \\ - national survey}

Bỉbliografie

DOI http://dx.doi.org/

10.1055/s-0042-101157

Online-Publikation: 13.5.2016

Gesundheitswesen 2016;

78 (Suppl. 1): e97-e102

(c) Georg Thieme Verlag KG

Stuttgart · New York

ISSN 0949-7013

Korrespondenzadresse

Antonius Reifferscheid

Diplom Gesundheitsökonom

Lehrstuhl für Medizinmanagement

Universität Duisburg-Essen

Schützenbahn 70

45127 Essen

antonius.reifferscheid@

medman.uni-due.de

\section{Zusammenfassung \\ $\nabla$}

Ziel der Studie: Gegenwärtig schreibt fast jedes zweite Krankenhaus Verluste. Aufgrund dieser schwierigen wirtschaftlichen Rahmenbedingungen sind in der Pflege die Personalzahlen zuletzt nicht adäquat an die Fallzahlsteigerungen angepasst worden. Mehrere aktuelle Befragungen von Pflegekräften weisen auf die gestiegenen Arbeitsanforderungen und daraus resultierende Versorgungsdefizite in der Pflege hin. Ziel dieser Studie war es zu untersuchen, ob die Leitungspersonen im Krankenhaus (Pflegedienstleitungen, Chefärzte und Geschäftsführer) aus ihrer jeweiligen Perspektive diese Problematik in vergleichbarer Form bestätigen.

Methodik: Zunächst wurden halbstrukturierte Interviews mit Chefärzten, Pflegedienstleitungen, Geschäftsführern und Trägern geführt. Auf Basis der qualitativen Erkenntnisse wurden berufsgruppenspezifische Fragebögen entwickelt, die im Frühjahr 2014 an knapp 5000 Leitungspersonen verschickt wurden.

Ergebnisse: Es konnte ein Rücklauf von insgesamt $43 \%$ erzielt werden. Alle Leitungspersonen bestätigen einen erheblichen wirtschaftlichen Druck, der sich spürbar auf die Patientenversorgung auswirkt - insbesondere bei der Pflege und der menschlichen Zuwendung werden erhebliche Defizite wahrgenommen. Der Großteil der Pflegedienstleitungen ist der Ansicht, dass dem Pflegepersonal eigentlich nicht genügend Zeit für Verfügung steht, um alle notwendigen Maßnahmen durchzuführen. Als Ursache für die Vorenthaltung von notwendigen Pflegeleistungen ist insbesondere die Personalknappheit zu sehen. Diese unzureichende Personalausstattung ist allerdings nicht nur auf die finanziellen Rahmenbedingungen, sondern auch auf den zunehmend spürbaren Fachkräftemangel in der Pflege zurückzuführen.

Schlussfolgerung: Insgesamt werden von allen Leitungspersonengruppen im pflegerischen Be-

\section{Abstract \\ $\nabla$}

Introduction: Currently, almost every second hospital is in financial deficit. Because of financial restrictions in the nursing profession, the staff has not been adequately adjusted to the increasing case volume. Current studies emphasize the rising workload of nursing staff and the resulting deficits in patient care. The aim of this study was to research whether the supervisory staff (nursing directors, chief physicians and hospital managers) also perceives these problems.

Method: First, semi-structured interviews with chief physicians, nursing directors, hospital managers and hospital owners were conducted. Based on these qualitative insights, occupational group-specific questionnaires were developed. In spring 2014, these were sent to almost 5000 supervisors.

Results: There was a response rate of $43 \%$. All respondents confirmed considerable economic restrictions related to the patient care - the greatest deficits were perceived concerning nursing care and personal attention given to patients. Moreover, the nursing directors were of the opinion that the nursing staff did not have enough time to perform all the necessary services. In particular, inadequate staffing was seen to be the cause of the rationing of nursing services. However, not only financial constraints but also a shortage of skilled staff increased the likelihood of withholding nursing services.

Conclusion: In sum, all supervisory groups perceived large deficits in nursing care. With the current staff levels, nursing directors can hardly ensure provision of all necessary nursing services. The nurse support program will improve this situation slightly. In the medium term, the implementation of other instruments is necessary. 
reich die größten Versorgungsdefizite wahrgenommen. Mit der gegenwärtigen pflegerischen Personalausstattung kann die Erbringung aller erforderlichen Leistungen nach Ansicht der Pflegedirektoren nicht immer gewährleistet werden. Während das Pflegeförderprogramm die Personalsituation kurzfristig leicht entspannen wird, sind mittelfristig andere Instrumente erforderlich.

\section{Einleitung \\ $\nabla$}

Gegenwärtig stehen die deutschen Krankenhäuser unter einem hohen finanziellen Druck. Als eine wesentliche Ursache hierfür ist die unzureichende Investitionsförderung der Bundesländer zu sehen, die (inflationsbereinigt) seit Jahrzehnten zurückgeht. Angesichts eines Investitionsstaus von ca. 15 Mrd. € [1] werden die Krankenhäuser zunehmend gezwungen, notwendige Investitionen selber zu finanzieren. Die hierfür erforderlichen Mittel müssen zum Großteil aus den DRG-Erlösen generiert werden, obwohl diese lediglich die durchschnittlichen Behandlungskosten abdecken. Angesichts dieser Rahmenbedingungen ist es wenig verwunderlich, dass gegenwärtig 40-50\% der Krankenhäuser Verluste schreiben [1,2].

Zur Verbesserung der wirtschaftlichen Situation bieten sich den Krankenhäusern 2 grundsätzliche Strategien. Entweder sie senken die Kosten durch weitere Rationalisierungsmaßnahmen oder erhöhen die Erlöse über Fallzahlsteigerungen. Vor allem der pflegerische Bereich erschien in den letzten Jahren von der Kombination aus Kostenbegrenzung und Fallzahlsteigerung betroffen. Im Vergleich zur Fallzahlsteigerung im Zeitraum 20042012 von insgesamt $11 \%$ hat sich die Personalausstattung im pflegerischen Bereich (trotz eines Pflege-Förderprogramms von 2009-2011) mit 3\% bzw. 10000 zusätzlichen Stellen eindeutig unterdurchschnittlich entwickelt, während bspw. die ärztliche Personalausstattung mit $25 \%$ mehr Vollkräften deutlich überproportional gestiegen ist [3]. Ein Grund hierfür könnte sein, dass die Pflege keine Erlösrelevanz im DRG-System besitzt. Zwar wird mit der Pflegepersonalregelung (PPR) ein Instrument zur Messung des Pflegepersonalbedarfs bei der Kalkulation der DRGs berücksichtigt - dies dient jedoch (nur) dazu, das vorhandene Personal kalkulatorisch auf die einzelnen DRGs zu verteilen. Insofern ist die Ausfinanzierung einer bedarfsgerechten Pflege nicht zwangsläufig gewährleistet. Auch der Pflegekomplexmaßnahmen-Score (PKMS), der über pflegebezogene Zusatzentgelte zur adäquaten Vergütung hochaufwändiger Pflegeleistungen beitragen sollte, änderte diese Situation aufgrund hohem Dokumentationsaufwand und begrenzter Anwendbarkeit kaum $[4,5]$.

\section{Versorgungsdefizite in der Pflege}

Diverse Studien weisen auf gestiegene Arbeitsanforderungen und die daraus resultierenden Versorgungsdefizite in der Pflege hin. Im internationalen Vergleich ist vorrangig festzustellen, dass in Deutschland verhältnismäßig wenig Pflegekräfte eingesetzt werden. So werden von einer Pflegekraft durchschnittlich 10,5 Patienten pro Schicht betreut (bei ausschließlicher Betrachtung der qualifizierten Pflegekräfte sind es 13 Patienten), was im internationalen Vergleich von 12 Ländern den schlechtesten Wert darstellt [6]. Dies ist insbesondere vor dem Kontext zu sehen, dass das Mortalitätsrisiko mit jedem zusätzlichen zu betreuenden Patienten je Pflegekraft statistisch um 7\% steigt [7].
Auch wenn bei der Pflege bereits mit der Aufhebung der PPR ein Personalabbau beobachtet werden konnte [8], wurde mit der Einführung des DRG-Systems ein weiterer Anstieg der quantitativen Anforderungen im administrativen Bereich und ein Rückgang patientennaher Tätigkeiten festgestellt $[9,10]$. Vor allem bei der Betreuung schwerkranker Patienten mit hohem Betreuungsaufwand werden von den Pflegekräften oft Mängel in der Versorgung berichtet [11]. Gerade in personalaufwändigen Bereichen (Geriatrie, Intensivstationen) wird häufig eine unzureichende Besetzung festgestellt, was die Unterlassung notwendiger Pflegeleistungen, eine unzureichende Beobachtung sowie die Durchführung vermeidbarer Zwangsmaßnahmen zur Folge hat $[12,13]$.

Eine weitere aktuelle Befragung von Pflegekräften zeigt auf, dass über $80 \%$ der Pflegekräfte im Arbeitsalltag Patientengespräche rationieren [14] und bestätigt damit frühere Forschungsergebnisse [15]. Neben Problemen bei der persönlichen Zuwendung werden aber auch von jeder fünften Pflegekraft Defizite in der Patientenlagerung sowie der Medikamentengabe eingeräumt. Als Ursachen werden in diesem Zusammenhang die unzureichende Personalausstattung und organisatorische Aspekte identifiziert. Gleichzeitig wird herausgestellt, dass bezüglich der impliziten Rationierung von Pflegeleistungen ein erheblicher nationaler Forschungsbedarf besteht [14].

Außerdem treten bei der pflegerischen Versorgung verstärkt Produktivitätserwägungen in den Vordergrund [16]. Interessanterweise haben die Patienten noch häufiger als die Pflegekräfte das Gefühl, dass ihre Behandlung auch von wirtschaftlichen Erwägungen mitbestimmt wird. In dem Zusammenhang sehen nur noch 59\% der Pflegekräfte die zwischenmenschliche Zuwendung als grundsätzliches Element der pflegerischen Versorgung an [17].

Die angesprochenen Forschungsarbeiten zeigen insgesamt erhebliche Defizite in der pflegerischen Versorgung auf. Gleichzeitig ist darauf hinzuweisen, dass die Befragungen weitgehend in der operativ tätigen Pflege (Pflegekräfte/Stationsleitungen) durchgeführt wurden. Insofern besteht ein Forschungsdefizit hinsichtlich der Wahrnehmung der aktuellen Problemlagen aus Sicht der Leitungskräfte (Pflegedienstleitungen bzw. Pflegedirektoren (PD)). Weiterführend hat auch nur eine Untersuchung [17] eine Triangulation der Ergebnisse durch zusätzliche Befragung von Patienten und Ärzten umgesetzt und dabei relevante Unterschiede in der Wahrnehmung der Befragtengruppen festgestellt. Auch in dieser Studie werden die Antworten der PD vor dem Hintergrund der Sichtweise der ärztlichen und der kaufmännischen Leitungspersonen eingeordnet. Von besonderem Interesse ist in diesem Zusammenhang, ob die bislang beobachteten pflegerischen Versorgungsdefizite auch von anderen Berufsgruppen registriert werden.

\section{Methodik}

$\nabla$

Um einen thematischen Überblick über potentiell interessante Fragestellungen $\mathrm{zu}$ gewinnen wurden neben der Sichtung der wissenschaftlichen Literatur 12 leitfadengestützte Interviews mit verschiedenen Leitungspersonen (PD, Chefärzte (CÄ), Geschäftsführer (GF) und Träger) durchgeführt. Zentrale Inhalte der Gespräche waren die wirtschaftlichen Rahmenbedingungen, der Umgang mit Mittelknappheit und die daraus resultierenden Konsequenzen im Hinblick auf die Patientenversorgung. Die Inter- 
views wurden im Anschluss inhaltsanalytisch ausgewertet [18]. Basierend auf den Erkenntnissen der Literatur und der Interviews wurden berufsgruppenspezifische Fragebögen für PD, CÄ und GF entwickelt. Die für diesen Artikel relevanten Fragebogen-Items sind online unter http://udue.de/FragebogenPD einsehbar.

Insgesamt wurden im Frühjahr 2014 im Rahmen des Forschungsprojektes „Umgang mit Mittelknappheit im Krankenhaus“ knapp 5000 Fragebögen postalisch versendet. Hierbei wurden 865 PD, 3163 CÄ und 869 GF angeschrieben, die über die Qualitätsberichte identifiziert wurden. Krankenhäuser mit weniger als 100 Betten wurden hierbei nicht beachtet. Auf einen Verknüpfungsversuch der Antworten der Leitungspersonen auf Krankenhausebene wurde a priori zwecks Gewährleistung einer größtmöglichen Anonymität verzichtet.

Neben einer deskriptiven Beschreibung der Antworten wurde die Frage zur Vorenthaltung pflegerischer Leistungen auf ihre Einflussfaktoren untersucht. Die Antworten der Befragten, die auf ordinalem Niveau vorlagen, wurden zunächst dichotomisiert und im Anschluss mittels einer binär logistischen Regression analysiert. Für dieses multiple Analyseverfahren wurden ausschließlich die Antworten der PD genutzt - aufgrund fehlender Angaben wurden 30 Fragebögen ausgeschlossen. Die statistische Auswertung erfolgte im Statistikprogramm SPSS 20.

\section{Ergebnisse}

Insgesamt haben 396 PD, 1432 CÄ und 284 GF den jeweiligen Fragebogen beantwortet, was einer Rücklaufquote von insgesamt $43 \%$ entspricht (PD: $46 \%$; CÄ: $45 \%$, GF: 33\%). Fast jeder zweite Befragte arbeitet in einem Krankenhaus mit einem defizitären Jahresabschluss, was den in der Literatur genannten Zahlen entspricht [1,2]. Im Hinblick auf die Krankenhausgröße und die Trägerschaft ist festzustellen, dass größere Krankenhäuser und öffentliche bzw. freigemeinnützige Krankenhäuser leicht überproportional vertreten sind.
In der Befragung zeigt sich, dass von sämtlichen Befragten ein großer wirtschaftlicher Druck wahrgenommen wird. Gerade die PD und GF spüren die ökonomischen Rahmenbedingungen, sodass 93 bzw. 95\% von einem deutlichen bis starken finanziellen Druck berichten. Bei den CÄ wird die Situation marginal besser bewertet. Dieser Druck manifestiert sich insofern, dass $72 \%$ der PD „oft“ oder „immer“ Entscheidungskonflikte zwischen pflegerischen und ökonomischen Zielsetzungen wahrnehmen, während nur 45\% der CÄ häufige Entscheidungskonflikte angeben. Angesichts dieser Ausgangssituation ist es wenig überraschend, dass 37\% der PD die personelle Ausstattung in der Pflege als "schlecht" oder "sehr schlecht“ bewerten - umgekehrt bewerten nur $17 \%$ der PD mit „gut“ oder „sehr gut“ ( $\bullet$ Abb. 1). Diese Einschätzung wird von den CÄ weitgehend bestätigt. Nur die GF sehen die Situation in der Pflege positiver. Fast jeder zweite GF spricht von einer guten Pflegepersonalausstattung im eigenen Krankenhaus. Die technische Ausstattung und die ärztlichen Personalbesetzung werden insgesamt deutlich positiver wahrgenommen.

Defizite in der personellen Ausstattung der Pflege sind jedoch nicht ausschließlich den wirtschaftlichen Rahmenbedingungen zuzuschreiben. Ebenfalls zeigt der sich zunehmend abzeichnende Pflegefachkräftemangel bereits erste Auswirkungen. 56\% der PD berichten bereits von Stellenbesetzungsproblemen, davon aber nur 6\% von aktuell größeren Schwierigkeiten bei der Personalgewinnung. Sowohl aus finanziellen Gründen aber auch, um der Knappheit an qualifizierten Pflegepersonal entgegenzuwirken, setzen 95\% der PD Pflegehilfskräfte und Servicekräfte ein $18 \%$ der PD setzen hierbei bereits intensiv auf diese Berufsgruppen. Mittelfristig wird deren Bedeutung weiter steigen. Fast zwei Drittel (66\%) der PD geben an, künftig verstärkt auf Pflegehilfskräfte und Servicekräfte zurückgreifen zu müssen bzw. zu wollen.

Die verschiedenen Leitungspersonen sind sich weitgehend einig, dass die gegenwärtigen wirtschaftlichen Rahmenbedingungen letztendlich auch spürbare Konsequenzen für die Patienten im-

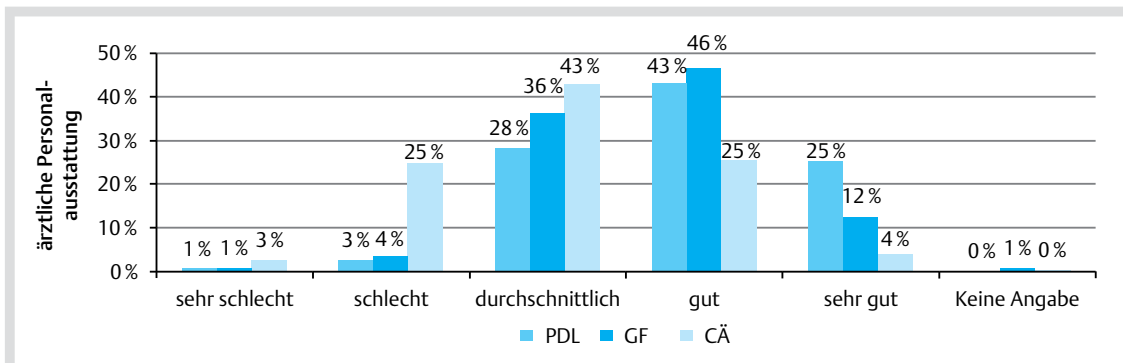

Abb. 1 Bewertung der Strukturqualität („Wie bewerten Sie die ärztliche Personalausstattung/ pflegerische Personalausstattung/technische Ausstattung in Ihrem Krankenhaus?").
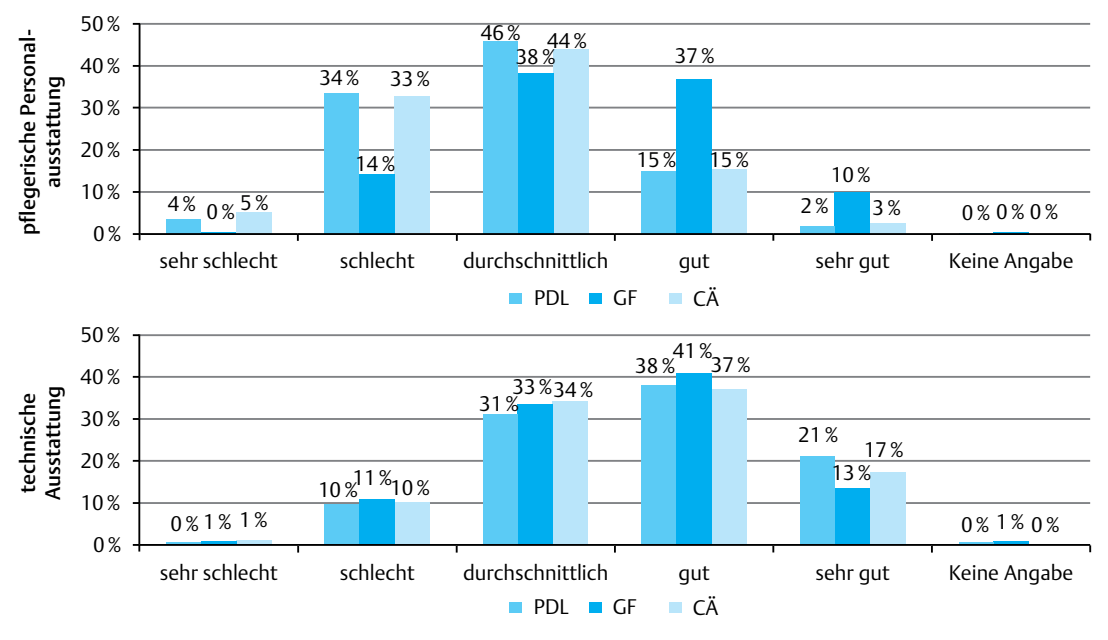


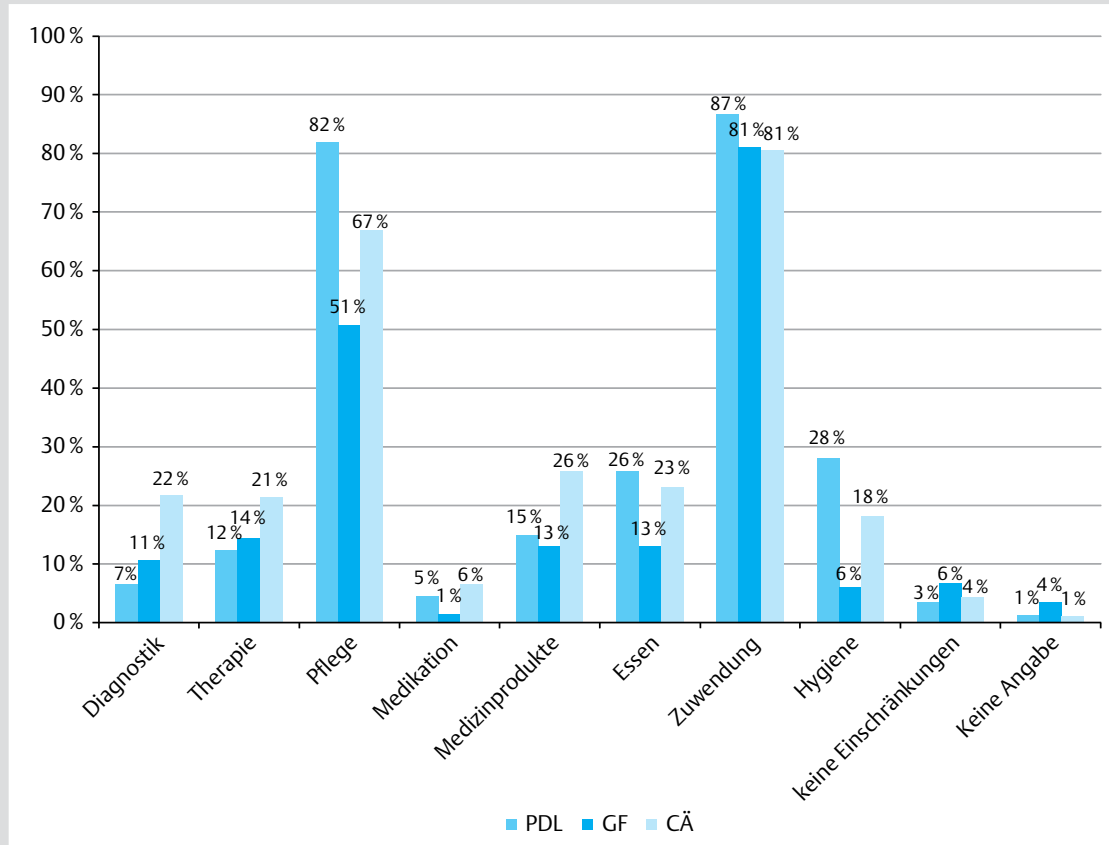

Abb. 2 Versorgungsdefizite aufgrund wirtschaftlicher Rahmenbedingungen (,In welchen Bereichen sehen Sie spürbare Einschränkungen der Patientenversorgung?“).

Tab. 1 Ergebnisse der binär logistischen Regression zu den Einflussfaktoren von Rationierung in der Pflege.

\begin{tabular}{|c|c|c|c|c|c|c|c|}
\hline \multirow[t]{3}{*}{ Einflussfaktoren } & \multirow{3}{*}{$\begin{array}{l}\text { nicht stan- } \\
\text { dardisierte } \\
\text { Koeffizienten }\end{array}$} & \multirow[t]{3}{*}{$\begin{array}{l}\text { Standard- } \\
\text { fehler }\end{array}$} & \multirow[t]{3}{*}{$\begin{array}{l}\text { Odds } \\
\text { Ratio }\end{array}$} & \multicolumn{2}{|c|}{$\begin{array}{l}95 \% \text { Konfidenzintervall } \\
\text { für Odds Ratio }\end{array}$} & \multirow[t]{3}{*}{ p-Wert } & \multirow{3}{*}{$\begin{array}{l}\text { standardi- } \\
\text { sierte Koef- } \\
\text { fizienten }\end{array}$} \\
\hline & & & & Unterer & Oberer & & \\
\hline & & & & Wert & Wert & & \\
\hline $\begin{array}{l}\text { hoher wahrgenommener wirtschaftlicher Druck ( } 1 \text { =ja; } \\
0=\text { nein) }\end{array}$ & 1,589 & 0,539 & 4,897 & 1,703 & 14,086 & $0,003^{*}$ & 0,064 \\
\hline positiver Jahresabschluss 2013 des KH ( $1=$ ja; 0 = nein) & 0,537 & 0,357 & 1,711 & 0,849 & 3,448 & 0,133 & 0,040 \\
\hline schlechte ärztliche Ausstattung der $F A B(1=\mathrm{ja} ; 0=$ nein $)$ & 0,773 & 1,143 & 2,167 & 0,230 & 20,373 & 0,499 & 0,022 \\
\hline schlechte pflegerische Ausstattung der FAB ( $1=$ ja; $0=$ nein $)$ & 0,919 & 0,414 & 2,506 & 1,113 & 5,641 & $0,026^{*}$ & 0,071 \\
\hline schlechte technische Ausstattung der FAB ( $1=$ ja; $0=$ nein $)$ & 0,508 & 0,611 & 1,662 & 0,502 & 5,501 & 0,405 & 0,023 \\
\hline häufige Entscheidungskonflikte ( 1 = ja; 0 = nein) & 1,349 & 0,328 & 3,854 & 2,028 & 7,323 & $<0,001^{*}$ & 0,096 \\
\hline Stellenbesetzungsprobleme in der Pflege $(1=\mathrm{ja} ; 0=$ nein $)$ & 0,668 & 0,316 & 1,951 & 1,051 & 3,622 & $0,034^{*}$ & 0,053 \\
\hline $\begin{array}{l}\text { weitere wahrgenommene Rationialisierungsmöglichkeiten in } \\
\text { Pflege ( } 1=\text { ja; } 0=\text { nein })\end{array}$ & $-0,825$ & 0,337 & 0,438 & 0,226 & 0,848 & $0,014^{*}$ & $-0,056$ \\
\hline großes $\mathrm{KH}$ mit $>400$ Betten ( $1=$ ja; $0=$ nein $)$ & 0,588 & 0,334 & 1,801 & 0,936 & 3,464 & 0,078 & 0,046 \\
\hline Freigemeinnütziger Träger ( $1=\mathrm{ja} ; 0=$ nein $)$ & $-0,086$ & 0,354 & 0,918 & 0,458 & 1,838 & 0,809 & $-0,007$ \\
\hline Privater Träger ( $1=$ ja; $0=$ nein $)$ & $-0,786$ & 0,514 & 0,456 & 0,166 & 1,249 & 0,127 & $-0,040$ \\
\hline KH Teil eines Verbundes $(1=\mathrm{ja} ; 0=$ nein $)$ & $-0,133$ & 0,341 & 0,875 & 0,448 & 1,709 & 0,697 & $-0,010$ \\
\hline Konstante & $-1,451$ & 0,577 & 0,234 & & & $0,012^{*}$ & \\
\hline
\end{tabular}

Nagelkerkes $\mathrm{R}^{2}=0,333^{*}$ signifikant auf dem Niveau $\alpha=0,05$

plizieren. $82 \%$ der PD, $71 \%$ der CÄ und 66\% der GF sind tendenziell davon überzeugt, dass sich der finanzielle Druck auch auf die Patientenversorgung auswirkt. Gerade die Pflege und die persönliche Zuwendung werden dabei eindeutig als Hauptproblembereiche identifiziert ( $\odot$ Abb. 2). So sehen in der Pflege $82 \%$ der PD, 67\% der CÄ und 51\% der GF Defizite. Bei der persönlichen Zuwendung zum Patienten ist das Antwortverhalten noch klarer ausgeprägt - so berichten $87 \%$ der PD und jeweils $81 \%$ der CÄ und GF von Problemen in diesem Bereich. Als weitere Probleme werden von den Leitungspersonen Defizite bei der Hygiene und der Essensversorgung benannt. Darüber hinaus werden von jedem fünften CÄ Einschränkungen bei Diagnostik, Therapie und der Versorgung mit Medizinprodukten gesehen.

Die beobachteten Defizite bei der Pflege und der Zuwendung wurden näher untersucht, mit besonderem Fokus auf der Rationierung in der Pflege. Rationierung wird hierbei als Vorenthal- tung notwendiger pflegerischer Maßnahmen verstanden. Die PD wurden daher explizit gefragt: „Glauben Sie, dass Ihrem pflegerischen Personal immer ausreichend Zeit zur Verfügung steht, um den Patienten alle notwendige Pflegeleistungen zukommen zu lassen?" Insgesamt 79\% der PD verneinen dies tendenziell. Somit gehen die PD mit überraschender Deutlichkeit davon aus, dass es in ihren Krankenhäusern zumindest gelegentlich zur Rationierung von Pflegeleistungen kommt.

\section{Ursachen der Rationierung im pflegerischen Bereich}

Die Ergebnisse des binär-logistischen Regressionsmodells, das die Einflussfaktoren der wahrgenommenen Rationierung in der Pflege untersucht, sind $\odot$ Tab. 1 zu entnehmen. In Krankenhäusern, in denen ein hoher Druck der wirtschaftlichen Rahmenbedingungen wahrgenommen wird, werden Pflegeleistungen eher vorenthalten. Darüber hinaus führt eine schlechte Personalbe- 
setzung häufiger zu einer Rationierung von Leistungen. Die Besetzung im ärztlichen Dienst, die technische Ausstattung sowie das finanzielle Ergebnis des Krankenhauses besitzen hingegen keinen signifikanten Einfluss. Gleichzeitig ist Rationierung in Einrichtungen stärker verbreitet, in denen häufig Entscheidungskonflikte zwischen pflegerischen und wirtschaftlichen Zielsetzungen wahrgenommen werden. Dort, wo PD oft mit solchen Konflikten konfrontiert sind, kommt es in Folge eher zur Unterlassung notwendiger Pflegemaßnahmen. Darüber hinaus hängt die Rationierungsproblematik ebenfalls damit zusammen, ob das Krankenhaus Probleme bei der Besetzung von Pflegestellen hat. Dies weist darauf hin, dass nicht alleine wirtschaftliche Rahmenbedingungen zur Leistungsvorenthaltung beitragen, sondern der sich abzeichnende Fachkräftemangel in der Pflege bereits heute die Patientenversorgung negativ beeinträchtigt. Ferner haben wahrgenommene potentielle Effizienzreserven einen signifikanten Einfluss auf die Vorenthaltung pflegerischer Leistungen. Dort wo größere Wirtschaftlichkeitspotenziale vermutet werden, stellt die pflegerische Leistungsvorenthaltung ein geringeres Problem dar. Zudem ist auffällig, dass die Krankenhausgröße keinen signifikanten Effekt zeigt. Gleichzeitig zeigen auch die Trägerschaft und die Zugehörigkeit zu einem Krankenhausverbund keinerlei Einfluss auf die wahrgenommene Rationierung in der Pflege.

\section{Weitere Konsequenzen}

Aufgrund der knappen Personalbesetzung, sind 88\% der PD, 72\% der CÄ und $81 \%$ der GF der Meinung, dass Ärzten und Pflege nur „selten“ bis „manchmal“ ausreichend Zeit für die persönliche Zuwendung zum Patienten zur Verfügung steht. Angesichts der Bewertung der gegenwärtigen Versorgungssituation sind sich PD und GF weitgehend einig, dass der pflegerische Bereich kaum weitere Rationalisierungspotenziale bietet. 75\% der PD und 61\% der GF vermuten in diesem Bereich kaum weitere relevante Einsparmöglichkeiten. Zuletzt ist darauf hinzuweisen, dass die aktuelle Situation zu einer gewissen Unzufriedenheit gegenüber dem DRG-System führt. 68\% der PD und 71\% der CÄ vertreten eine eher negative Einstellung gegenüber dem DRG-System - bei den GF sind es nur $24 \%$.

\section{Diskussion}

\section{$\nabla$}

Bei dieser Studie sind verschiedene Limitationen zu beachten. So ist darauf hinzuweisen, dass die Einflussfaktoren von pflegerischer Rationierung nicht kausal abgesichert werden konnten. Zu diesem Zweck wären weitere Untersuchungen mit mehreren Erhebungszeitpunkten wünschenswert. Auch organisatorische Aspekte (z.B. Führungsfähigkeiten der Stationsleitung) konnten aufgrund der Leitungsperspektive nicht wirklich abgebildet werden, auch wenn dies weitere (stationsbezogene) potentiell relevante Einflussfaktoren von Rationierung sind [14]. Darüber hinaus würde eine Verknüpfung der Antworten der Berufsgruppen auf Krankenhausebene eine noch höhere Aussagekraft gewährleisten. Zugunsten einer größtmöglichen Anonymität und zur Gewährleistung eines höheren Rücklaufs wurde jedoch auf die Möglichkeit einer solchen Zuordnung verzichtet. Zuletzt ist darauf hinzuweisen, dass im Rahmen dieser Befragung nur die subjektive Wahrnehmung der Leitungspersonen erfasst werden konnte, die nicht immer mit der tatsächlichen Versorgungsrealität übereinstimmen muss. Ebenfalls ist nicht auszuschließen, dass Befragte bei der Beantwortung ggf. berufsbezogene Interessen verfolgen.

Trotz der verschiedenen Einschränkungen ist als zentrales Ergebnis festzuhalten, dass von allen Leitungsgruppen ein hoher wirtschaftlicher Druck wahrgenommen wird, die größten Probleme in der Patientenversorgung aber durchgängig dem pflegerischen Bereich zugeordnet werden. Dies wird ebenfalls durch die Einschätzung der PD zur Verbreitung von Rationierung der Pflegeleistungen untermauert. Auch wenn pflegerische Versorgungsdefizite von den PD am stärksten wahrgenommen werden, zeigt die weitgehende Übereinstimmung mit den anderen Befragtengruppen, dass diese gegenwärtigen Defizite auch außerhalb der Pflege deutlich wahrgenommen werden.

Auch im Hinblick auf den demografischen Wandel und den daraus resultierenden steigenden Pflegekräftebedarf durch ältere und multimorbide Patienten ist die aktuelle Entwicklung als problematisch anzusehen und gesundheitspolitischer Handlungsbedarf gegeben. Grundsätzlich sieht der Koalitionsvertrag neben dem Personalkostennachweis eine Prüfung vor, ob die Pflege in „ausreichender Höhe und Gewichtung [in den DRGs] berücksichtigt“ ist. In diesem Zusammenhang ist auch explizit auf die hohe Pflegevariabilität des Pflegeaufwands innerhalb der einzelnen DRGs hinzuweisen, die im Schweizer System festgestellt wurde [19]. Das neue Pflegeförderprogramm mit einem Gesamtvolumen von 660 Mio. € für einen 3-jahreszeitraum und der Pflegezuschlag von 500 Mio. € können sicherlich kurzfristig zu einer gewissen Entspannung beitragen. Mittelfristig sind jedoch weitergehende Maßnahmen in der Krankenhauspflege zu ergreifen - wie bspw. Personalstandards [8] oder eine Weiterentwicklung der DRG-Klassifikationskriterien [19].

\section{Danksagung \\ $\nabla$}

Unser Dank gilt den zahlreichen Teilnehmern der Befragung. Das Projekt „Umgang mit Mittelknappheit im Krankenhaus“, in dessen Rahmen diese Studie entstanden ist, wurde von der gemeinnützigen Dr. Jürgen Meyer Stiftung gefördert. Die inhaltliche wissenschaftliche Arbeit wurde durch die Förderung nicht beeinflusst.

Interessenkonflikt: Die Autoren geben an, dass kein Interessenkonflikt besteht.

\section{Literatur}

1 Augurzky B, Hentschker C, Krolop S et al. Krankenhaus Rating Report 2014 - Mangelware Kapital: Wege aus der Investitionsfalle. Heidelberg: medhochzwei; 2014

2 Blum K, Löffert S, Offermanns $M$ et al. Krankenhaus Barometer 2013. Düsseldorf: Deutsches Krankenhaus Institut; 2013

3 Thomas D, Reifferscheid A, Pomorin $N$ et al. Instrumente zur Personalbemessung und -finanzierung in der Krankenhauspflege in Deutschland. IBES Diskussionsbeitrag Nr. 204. Essen 2010

4 Bechtel P, Smerdka-Arhelger I. Pflege im Wandel gestalten - Eine Führungsaufgabe. Lösungsansätze, Strategien, Chancen. Berlin: Springer; 2012

5 Blum K, Löffert S, Offermanns $M$ et al. Krankenhaus Barometer 2012. Düsseldorf: Deutsches Krankenhaus Institut; 2012

6 Aiken LH, Sermeus W, van den Heede K et al. Patient safety, satisfaction, and quality of hospital care: cross sectional surveys of nurses and patients in 12 countries in Europe and the United States. BMJ 2012; 344: e1717-e1730

7 Aiken LH, Sloane DM, Bruyneel L et al. Nurse staffing and education and hospital mortality in nine European countries: a retrospective observational study. Lancet 2014; 383: 1824-1830 
8 Simon M. Personalabbau im Pflegedienst der Krankenhäuser: Hintergründe, Ursachen, Perspektiven. Pflege \& Gesellschaft 2009; 14: 101-123

9 Schmidt S, Bartholomeyczik S, Dieterle WE et al. Arbeitsbedingungen für die Pflege in Krankenhäusern als Herausforderung. Pflege \& Gesellschaft 2008; 13: 102-118

10 Bartholomeyczik S. Reparaturbetrieb Krankenhaus, DRGs und ihre Auswirkungen aus Sicht der Pflege. Dr. med. Marbuse 2007; 166: 57-60

11 Isfort M, Weidner F, Gehlen D. Pflege-Thermometer 2009. Köln: Institut für angewandte Pflegeforschung e.V.; 2010

12 Isfort M, Weidner F, Gehlen D. Pflege-Thermometer 2012. Köln: Institut für angewandte Pflegeforschung e.V.; 2012

13 Isfort M, Klostermann J, Gehlen D et al. Pflege-Thermometer 2014. Köln: Institut für angewandte Pflegeforschung e.V.; 2014

14 Zander B, Dobler L, Bäumler $M$ et al. Implizite Rationierung von Pflegeleistungen in deutschen Akutkrankenhäusern. Gesundheitswesen 2014; 76: 727-734
15 Slotala L, Bauer U. „Das sind bloß manchmal die fünf Minuten, die fehlen." Pflege zwischen Kostendruck, Gewinninteressen und Qualitätsstandards. Pflege \& Gesellschaft 2009; 14: 54-66

16 Thomas D, Borchert $M$, Brockhaus $N$ et al. Dienstleistungsproduktivität in der Krankenhauspflege - Konzeptionelle Grundlagen einer Produktivitätsanalyse. Gesundheitswesen 2015; 77: e1-e7

17 Braun B, Buhr P, Klinke $S$ et al. Pauschalpatienten, Kurzlieger und Draufzahler - Auswirkungen der DRGs auf Versorgungsqualität und Arbeitsbedingungen im Krankenhaus. Bern: Huber; 2010

18 Mayring $P$. Qualitative Inhaltsanalyse, Grundlagen und Techniken. $11^{\text {th }}$ (ed.). Weinheim: Beltz; 2010

19 Baumberger $D$, Bürgin $R$, Bartholomeyczik S. Variabilität des Pflegeaufwands in den SwissDRG-Fallgruppen. Pflege 2014; 27: 105-115 